\title{
Ornithogalum Saponin OSW-1 Negatively Regulates Dendritic Cells to Enhance Immune Function
}

KE XIN ZHANG, XIN HE¹, JIA LI, J. YU², MEI GENG HU³, JUAN LU³ AND FENG QI FANG*

Department of Oncology, ${ }^{1}$ Department of Respiratory Medicine, The First Affiliated Hospital of Dalian Medical University, Dalian, Liaoning 116011, ²Department of Cancer Center, Affiliated Zhongshan Hospital of Dalian University, Dalian, Liaoning 116001, ${ }^{3}$ Development of Institute of Medicinal Plant, Chinese Academy of Medical Sciences, Peking Union Medical College, Beijing 100730, China

Zhang et al.: Ornithogalum Saponin OSW-1 Negatively Regulates Dendritic Cells

\begin{abstract}
Liliaceae plant Ornithogalum caudatum Jacq. is applied in the treatment of inflammation and tumors in ancient Chinese medicine. However, the impact of OSW-1 on immune function is not yet fully understood. Therefore, seeking and exploring effective intervention measures for the immune escape of tumor cells is essential for the prevention and treatment of cancer recurrence and metastasis. The aim of this study was intended to observe the process of OSW-1 on the immune function status of regulatory dendritic cells, which opened up new ideas for studying the anti-tumor mechanism of OSW-1. Our experimental results has confirmed that Ornithogalum saponin OSW-1 has a clear inhibitory effect on the expression of regulatory dendritic cell surface receptor, toll-like receptor 4 , blocking negative immune regulation of regulatory dendritic cells, promoting the proliferation of splenic lymphoid $T$ cells and enhancing immune activity, indicating that OSW-1 can exert an anti-tumor mechanism by regulating immunosuppression. Our research provides a new theoretical basis for the anti-tumor mechanism of OSW-1.
\end{abstract}

Key words: Ornithogalum saponin OSW-1, immunosuppression, toll-like receptor 4, T cells

Liliaceae plant Ornithogalum caudatum Jacq. is applied in the treatment of inflammation and tumors in ancient Chinese medicine. In 1992, the Japanese scholar Sashido extracted a saponin compound Orsaponin (OSW-1) from the rhizome of Ornithogalum for the first time ${ }^{[1-3]}$. A large number of studies have revealed that OSW-1 has a targeted anti-cancer effect and can directly kill cancer cells. Zhou et al. has found that the sensitivity of normal cells to OSW-1 is significantly lower than that of tumor cells and Half Maximal Inhibitory Concentration $\left(\mathrm{IC}_{50}\right.$ ) was 40 to 150 times that of tumor cells. OSW-1 plays an anti-tumor effect mainly through the inhibition on tumor cell growth and the promotion on cell apoptosis ${ }^{[4]}$. However, the impact of OSW-1 on immune function is not yet fully understood. regulatory Dendritic Cells (DCreg) are one subgroup of Dendritic Cells (DCs). They are the most powerful and the only antigen-presenting cells to activate naive $\mathrm{T}$ cells and stimulate the initial immune response known at present, as well as have the functions of inhibiting $\mathrm{T}$ cell proliferation, inducing regulatory $\mathrm{T}$ cell differentiation and supporting tumor immune tolerance and escape ${ }^{[5]}$. In recent years, the role of DCreg in tumor immunity has been paid more and more attention, inhibiting tumor immune escape, restoring $\mathrm{T}$ cell antitumor activity and restarting anti-tumor immunity, thus, regulating DCreg immune activity is expected to become an important target for the treatment of tumor immune escape ${ }^{[6]}$. The research on the mechanism that immune escape causes tumor cells to invade and migrate, and tumors cause immune tolerance and inhibit the anti-tumor immune effect is still in the preliminary stage. Therefore, seeking and exploring effective intervention measures for the immune escape of tumor cells is essential for the prevention and treatment of cancer recurrence and metastasis. This study intended to observe the process of OSW-1 on the immune function status of DCreg, which opened up new ideas for studying the anti-tumor mechanism of OSW-1. Instruments include Flow cytometer (BD FACSCalibur $^{\mathrm{TM}}$ flow cytometer, BD Biosciences, USA); microplate reader (Tecan M1000); MiniMACS magnetized cell sorter; SC-3610 low-speed centrifuge. Reagents used in the study are Ornithogalum saponin

*Address for correspondence

E-mail: ffqlj@163.com 
OSW-1 (Shanghai Ruichang Pharmaceutical Technology Co., Ltd., batch number: TDA9612), 3-(4,5-dimethylthiazol-2-yl)-2,5-diphenyl tetrazolium bromide (MTT), Biyuntian Bio; mouse dendritic cells

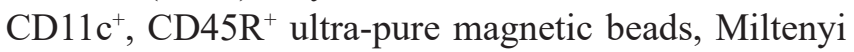
Biotec, Germany; Interleukin-12(IL-12), Interleukin-18 (IL-18) Enzyme-Linked Immunosorbent Assay (ELISA) kit, Beyotime Biotechnology company; TollLike Receptor 4 (TLR4) antibody, Abcam. Animals used in the study are Bagg Albino (BALB)/C mouse, male, 18 22 g, purchased from Beijing Huafukang Biotechnology Co., Ltd., with experimental unit license number: SYXK (J.) 2017-0020 and license number: SCXK (J.) 2019 -0008. Isolation and detection of mouse spleen DCreg (CD11 ${ }^{\text {low }}$ CD45RB ${ }^{\text {high }}$ DCs) is described in detail. BALB/C mice were sacrificed by removing the cervical vertebrae. After disinfecting the body surface with $75 \%$ ethanol, the spleen was aseptically removed on the ultra-clean workbench and washed in $4^{\circ}$ pre-cooled Phosphate Buffered Saline (PBS). The spleen was ground by the piston of a sterile syringe and passed through a 100 mesh filter. The cells were collected by centrifugation at $1500 \mathrm{rpm}$, resuspended in an appropriate amount of PBS and adjusted to $10 \times 10^{6}$ cells $/ \mathrm{ml}$ and subjected to magnetic sorting. After the mouse spleen-derived DCs were sorted by magnetic beads, the mini magnetic cell separation (miniMACS) immunomagnetic separation system was used to magnetically sort the DCs, the pelleted cells were resuspended in $1 \mathrm{ml}$ of buffer and the cell suspension was passed through the MACS magnetic separation column of which the negative part was CD11 $\mathrm{c}^{\text {low }} \mathrm{DC}$. And then CD11 $\mathrm{c}^{\text {low }} \mathrm{DC}$ was incubated with anti-mouse CD45RB immunomagnetic beads, and the positive part, namely CD11 ${ }^{\text {low }} \mathrm{CD} 45 \mathrm{RB}^{\text {high }} \mathrm{DCs}$, was separated and collected with the MiniMACS magnetized cell sorter. Cytotoxicity test of OSW-1 on DCreg is explained in detail. DCreg were seeded into a 96-well plate at a concentration of 8000 cells/well and an appropriate amount of the mother liquor containing OSW-1 was added, so that the final concentration was $6.25,12.5,25,50,100,200,400 \mu \mathrm{g} / \mathrm{ml}$, with 3 multiple holes for each concentration. Excipient dimethyl sulfoxide (DMSO) was added to blank control wells, with the final concentration of $0.5 \%$, incubated in an incubator with a $5 \% \mathrm{CO}_{2}$ at $37^{\circ}$ for $24 \mathrm{~h}$. Then $20 \mu \mathrm{l}$ of $5 \mathrm{mg} / \mathrm{ml}$ MTT was added to each well and continued to incubate for $4 \mathrm{~h}$. Then DMSO was added and the Optical Density (OD) value was measured at $570 \mathrm{~nm}$ using the microplate reader. The cell proliferation was characterized by the percentage of the OD value of the dosing hole and the control hole. Detection with ELISA method on the effect of OSW-1 on IL-12 and IL-18 levels secreted by DCreg is shown here. DCreg were seeded into a 96-well plate and an appropriate amount of mother liquor containing OSW-1 was added so that the final concentration was 25,50 and $100 \mu \mathrm{g} / \mathrm{ml}$, respectively. After incubation for $12 \mathrm{~h}$, the supernatant was centrifuged to measure the levels of IL-12 and IL18 by ELISA. The effect of OSW-1 on the proliferation of mouse spleen effector $\mathrm{T}$ cells is shown here. The spleen was aseptically separated for grinding and screening according to the method described above. After centrifugation at $1500 \mathrm{r} / \mathrm{min}$, an appropriate amount of PBS was added to form a cell suspension, lymphocyte separation solution (2 times the volume) was added along the tube wall and the supernatant was removed by centrifugation at $1500 \mathrm{r} / \mathrm{min}$ for $15 \mathrm{~min}$. The middle layer was aspirated using MiniMACS immunomagnetic separation system. Cluster of Differentiation $4 \quad\left(\mathrm{CD}^{+}\right) \quad \mathrm{T}$ lymphocytes were resuspended with $10 \%$ Fetal Calf Serum-Roswell Park Memorial Institute (FCS-RPMI) 1640 complete medium containing Concanavalin A (ConA), with the cell density adjusted to $2 \times 10^{6} / \mathrm{ml}$ and inoculated in a 96-well cell culture plate with $200 \mu \mathrm{l}$ per well. DCreg stimulated by OSW-1 at a concentration of 25, 50, 100 $\mu \mathrm{g} / \mathrm{ml}$ interacted with $\mathrm{T}$ lymphocytes in a $96-$ well plate at a ratio of 1:100 and they were placed in a cell culture incubator for $68 \mathrm{~h}$. MTT was added at $20 \mu \mathrm{l} /$ well and placed in the cell culture incubator for $4 \mathrm{~h}$. Then DMSO was added and the OD value was measured at $570 \mathrm{~nm}$. The cell proliferation was characterized by the percentage of the OD value of the dosing hole and the control hole. The mechanism of OSW-1 functions on DCreg is explained here. After the cells were processed according to the method aforesaid, the expression of TLR4 on the surface of the DCreg membrane was detected by flow cytometry and the expression level of molecule TLR4 was expressed by the average fluorescence intensity. Each of the above experiments was repeated three times under the same conditions. The experimental results were expressed as mean \pm standard error using GraphPad Prism 6.0 software. The $t$ test was used to analyze the significance of differences between groups and statistically significant differences are indicated as $\mathrm{p}<0.05$. Extraction and detection of mouse spleen DCreg $\left(\mathrm{CD} 11 \mathrm{c}^{\text {low }} \mathrm{CD} 45 \mathrm{RB}^{\text {high }} \mathrm{DCs}\right)$ was shown here. After normal mouse spleen mononuclear cells were sorted twice by MACS, the purity of CD11 $\mathrm{c}^{\text {low }} \mathrm{CD} 45 \mathrm{RB} \mathrm{B}^{\text {high }}$ DCs could reach more than $90 \%$, as shown in fig. 1 . 
OSW-1 in the dose range of $6.25,12.5,25,50,100 \mu \mathrm{g} /$ $\mathrm{ml}$ did not have a significant effect on the proliferation of DCreg, as shown in fig. 2. OSW-1 at concentrations of $200 \mu \mathrm{g} / \mathrm{ml}$ and $400 \mu \mathrm{g} / \mathrm{ml}$ significantly inhibited the proliferation of DCreg and 25, 50 and $100 \mu \mathrm{g} / \mathrm{ml}$ was used in subsequent experiments. The effect of OSW-1 on the levels of IL-12 and IL-18 secreted by DCreg is described here. As shown in fig. 3, the secretion of inflammatory cytokines (IL-12 and IL-18) of CD11 $\mathrm{c}^{\text {low }} \mathrm{CD} 45 \mathrm{RB}^{\text {high }} \mathrm{DCs}$ was significantly increased after OSW-1 stimulation, suggesting that OSW-1 could promote the negative regulation of $\mathrm{CD} 11 \mathrm{c}^{\text {low }} \mathrm{CD} 45 \mathrm{RB}^{\text {high }}$ DCs. The effect of OSW-1 on the proliferation of $\mathrm{T}$ lymphocytes stimulated by ConA through DCreg is described here. As shown in fig. 4, DCreg stimulated by OSW-1 at 25, 50 and $100 \mu \mathrm{g} / \mathrm{ml}$ interacted with $\mathrm{T}$ lymphocytes at a ratio of $1: 100$ for $3 \mathrm{~d}$. The proliferative response of splenic $\mathrm{T}$ lymphocytes to mitogen ConA stimulation was significantly enhanced $(p<0.01)$. The effect of OSW-1 on DCreg through TLR4 receptor is given below. As shown in fig. 5, after the intervention of OSW-1 at different concentrations, the fluorescence intensity of TLR 4 in the $50 \mu \mathrm{g} / \mathrm{ml}$ and $100 \mu \mathrm{g} / \mathrm{ml}$ groups decreased significantly, indicating that the OSW-1 could cause the downregulation of the expression level of the DCreg surface receptor TLR4. Invasion and metastasis are responsible for the death of $90 \%$ of cancer patients. Existing studies have shown that tumor cells evading immune surveillance through selfmutation, inducing $\mathrm{T}$ cell changes or relying on external factors is a very important mechanism related to cancer invasion and metastasis. The immune escape as well as invasion and migration of cancer cells are vital for the success or failure of treatment. DCs are the most powerful antigen-presenting cells in the body and they play an important role in the occurrence of some infectious diseases, tumors and autoimmune diseases. The negative regulatory ability of DCreg can induce the differentiation of $\mathrm{CD}^{+} \mathrm{T}$ lymphocytes to the $\mathrm{T}$ Helper 2 (Th2) cell type, ultimately mediating the body's immunosuppressive activity. Evidence suggests that TLR4 may be one of the main receptors for DCreg functional differentiation and participates in the negative immune regulation of DCreg. Negative immune regulation may be closely related to the invasion and migration of tumor cells, that is, negative immune regulation can promote the migration and survival of tumor cells; patients with negative immune regulation are more prone to metastasis. Activity tests demonstrate that the biological activity of Ornithogalum saponin OSW-1 is 10-100 times higher than that of several anticancer drugs currently in clinical use, such as etoposide, adriamycin and methotrexate. While showing strong cytotoxic activity to a variety of tumor cells, it has little toxicity to human normal cells $\left(\mathrm{IC}_{50}=1.51 \mu \mathrm{g} / \mathrm{ml}\right)$. Our experimental results has confirmed that Ornithogalum saponin OSW-1 has a clear inhibitory effect on the expression of DCreg surface receptor TLR4, blocking negative immune regulation of DCreg, promoting the proliferation of splenic lymphoid $\mathrm{T}$ cells and enhancing immune activity, indicating that OSW-1 can exert an anti-tumor mechanism by regulating immunosuppression. Accumulating treatments for immune escape are in preclinical and clinical trials. For example, Programmed Cell Death Protein 1 (PD-1) inhibitors, Cytotoxic T-Lymphocyte-Associated Protein 4 (CTLA4) inhibitors, etc., are all targeted therapeutic drugs related to inhibiting tumor immune escape in a broad sense. At present, immune escape causes tumor cells to invade and migrate, and tumors cause immune tolerance and inhibit the anti-tumor immune effect which is still in the preliminary stage. Therefore, seeking and exploring effective intervention measures for the immune escape of tumor cells is essential for the prevention and treatment of cancer recurrence and metastasis.
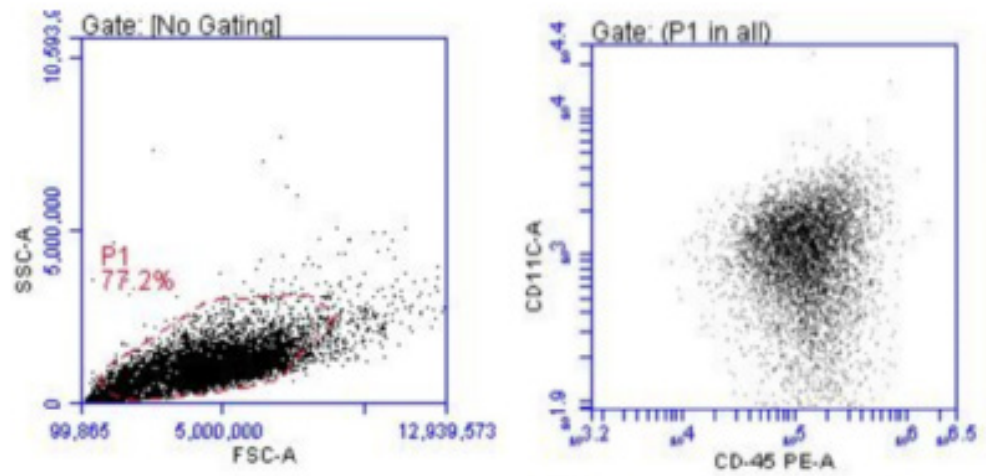

Fig. 1: Results of CD11 ${ }^{\text {low } C D 45 R B ~}{ }^{\text {high }}$ DCs in mouse spleen, OSW-1 to DCreg cytotoxicity test results 


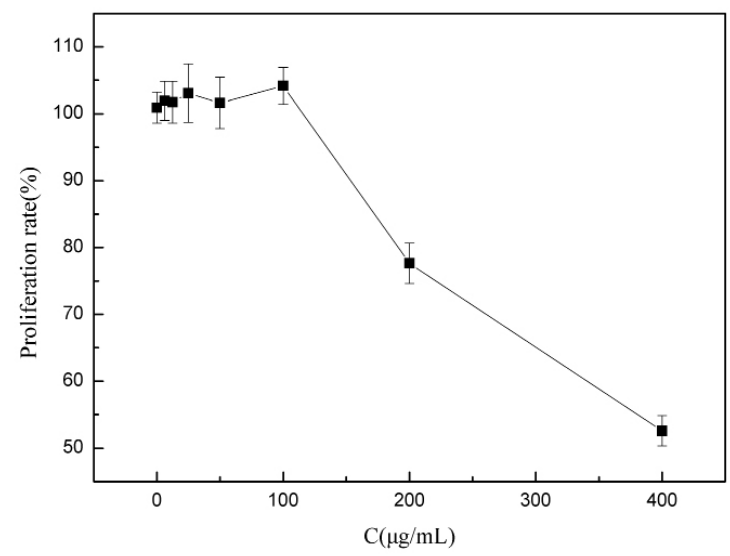

Fig. 2: The effect of OSW-1 at different concentrations on DCreg cytotoxicity

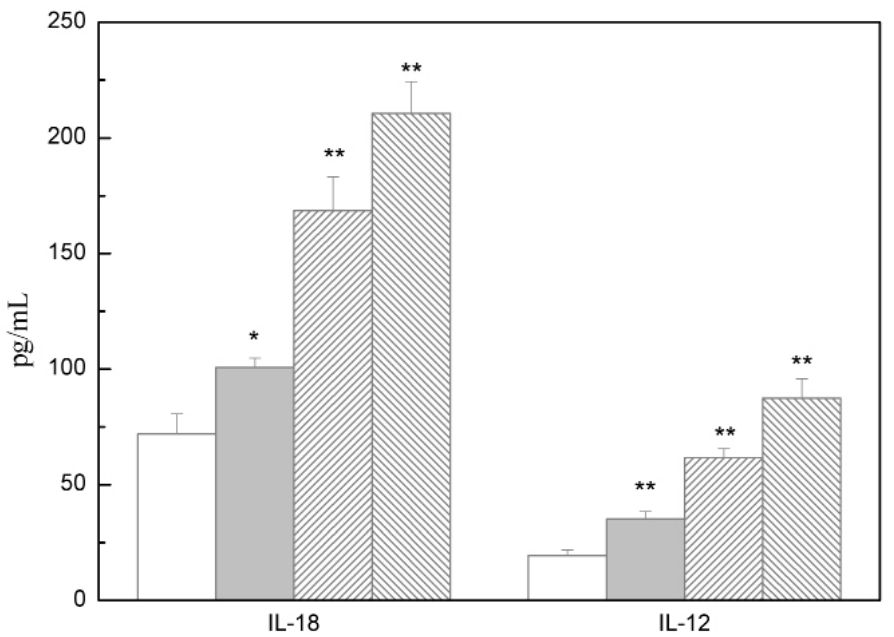

Fig. 3: The dose-effect relationship of the levels of IL-18 and IL-12 secreted by DCreg after OSW-1 stimulation (*p<0.05, versus

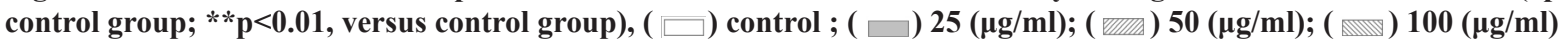

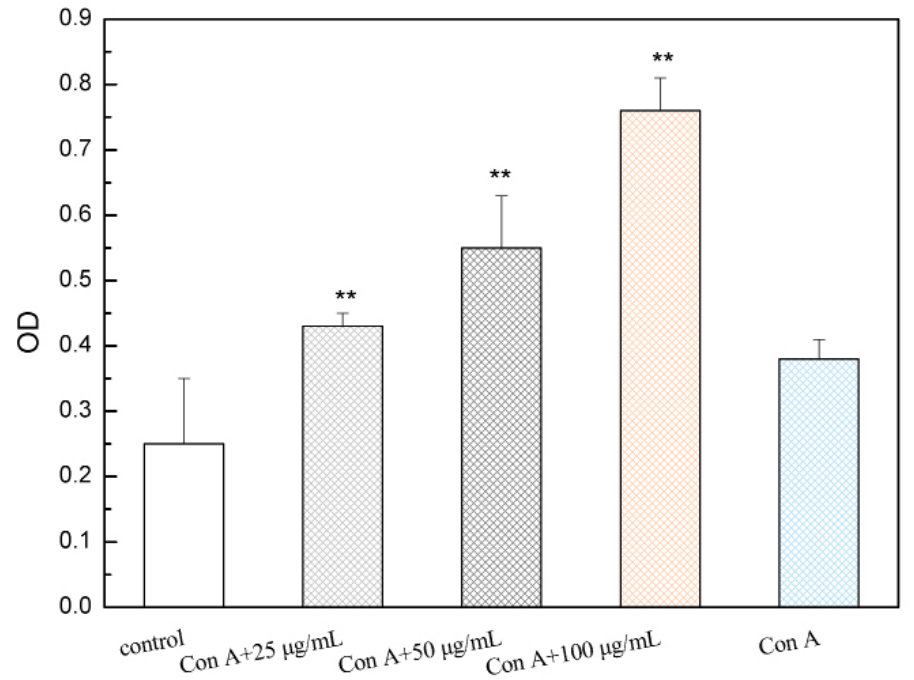

Fig. 4: The effect of DCreg stimulated by OSW-1 on the proliferative response of T lymphocytes $(* *$ p $<0.01$, versus ConA group) 


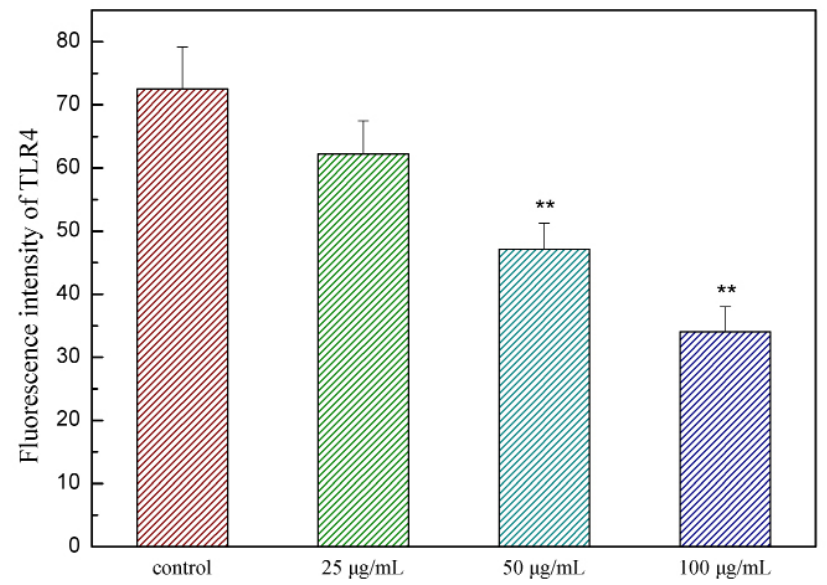

Fig. 5: Changes in TLR4 expression in cells in groups under different treatments

\section{Acknowledgements:}

This work was supported by the mechanism of high mobility group protein B1 influencing colon cancer cell invasion and migration through negative regulation of DCreg and the intervention effect of OSW-1 (No. 20170540263), Ke Xin Zhang and Xin He contributed equally to this work.

\section{Conflict of interests:}

The authors report no conflicts of interest.

\section{REFERENCES}

1. Xie MX, Zhou Z, Zou K. Research progress of Dieffenbachia saponin and its derivatives. China Pharm 2009;20(12):950-2.

2. Wang ZB, Wang W, Cheng KZ, Kong JQ. Research progress on the structure-activity relationship of Dieffenbachia saponin OSW-1. Chin Med Biotechnol 2013;(5):368-75.
3. Mei BS, Jin JC, Qian CS, Jin XL. Analysis of expression of miRNA spectrum in hepatoma cells affected by saponins extract of Ornithogalum saudersiae 2018;29(12):2910-5.

4. Zhou Y, Garcia-Prieto C, Carney DA, Xu RH, Pelicano H, Kang Y, et al. OSW-1: A natural compound with potent anticancer activity and a novel mechanism of action. J Natl Cancer Inst 2005;97(23):1781-5.

5. Liu BC, Yuan JL, Qiu Y. Research progress in the immunomodulatory effects of natural products on dendritic cells. Chin Herb Med 2018;49(5):1227-32.

6. Sun B, Wei Y, Shi B. Research progress on the classification of dendritic cells and the way of obtaining regulatory dendritic cells. Organ Transplant 2019;10(2):104-7.

This is an open access article distributed under the terms of the Creative Commons Attribution-NonCommercial-ShareAlike 3.0 License, which allows others to remix, tweak, and build upon the work non-commercially, as long as the author is credited and the new creations are licensed under the identical terms

This article was originally published in a special issue, "Novel Therapeutic Approaches in Biomedicine and Pharmaceutical Sciences" Indian J Pharm Sci 2021:83(6) Spl Issue "140-144" 